\title{
Die Rolle digitaler Bildungsprojekte in der Entwicklungszusammenarbeit
}

\begin{abstract}
Zusammenfassung
Im Spannungsfeld der einerseits bestehenden neuen Möglichkeiten und Herausforderungen der Digitalisierung und der andererseits noch geringen wissenschaftlichen Erforschung digitaler Projekte in der Entwicklungszusammenarbeit wird der Frage nachgegangen, welche Bedeutung die digitale Transformation für die Entwicklungszusammenarbeit hat. Im Artikel wird aufgezeigt, welche Rolle Digitalisierung in der Entwicklungszusammenarbeit, insbesondere im Kontext von Bildungsprojekten, einnimmt.
\end{abstract}

Schlüsselworte: Kommunikation, Soziale Medien, Bildung, Soziale Kompetenzen

\begin{abstract}
Between the poles of the new opportunities and challenges of digitalization and the low level of scientific monitoring of digital projects in development cooperation there is the question: „what kind of importance has digital transformation in the context of development cooperation?" The article analyzes which role the digitalization plays in development cooperation, especially in educational projects.
\end{abstract}

Keywords: Communication, social media, education, life skills

\section{Entwicklungen und Potentiale der Digitalisierung}

Bezüglich der aktuell in vielen Feldern stattfindenden Digitalisierung werden verstärkt Parallelen zur Industriellen Revolution gesehen und diskutiert. Grundlage der Argumentation für diese Parallelen ist, dass damals wie heute menschliche Fähigkeiten durch Technik ersetzt wurden beziehungsweise werden: Damals war es die Muskelkraft, die durch die Dampfmaschine in vielen Bereichen überflüssig wurde. Der Argumentation folgend gehen Tendenzen dahin, einige Fähigkeiten des menschlichen Gehirns durch die künstliche Intelligenz in ähnlichen Ausmaßen wie bei der Industriellen Revolution abzulösen (Schönig, Hoyer \& Pot- ratz, 2018, S. 158). Mit Blick auf den Trendradar_2030 (gut.org gemeinnützige Aktiengesellschaft betterplace lab, 2017) wird die Frage aufgeworfen, ob die künstliche Intelligenz und Big Data, 3D-Druck, das Internet der Dinge und neue Finanztechnologien das Potential haben unsere Welt zu verändern. Ob beispielsweise der vermehrte Einsatz von Robotern, die Massentauglichkeit von 3D-Druckern, das autonome Fahren von Kraftfahrzeugen und eine Verbreitung des digitalen Zahlungsverkehrs weltweit immer mehr Gegenwart als Zukunft werden, steht zur Diskussion (ebd.).

Neben diesen komplexen digitalen, wachsenden Trends steht eine bereits fortgeschrittene technische Entwicklung: Die Digitalisierung der Kommunikation. Eine niederschwellige Kommunikation kann durch den Zugang zu Mobiltelefonen, Computern und dem immer weiter verbreiteten Internetzugang möglich werden. Jedoch fehlt dieser Zugang noch für viele Menschen: Rund vier Milliarden Menschen weltweit haben keinen Zugang zum Internet und damit nicht ohne weiteres eine Chance an digitalen Neuentwicklungen teilzuhaben (The World Bank, 2016a, S. 4). Die Ausstattung mit Mobiltelefonen ist deutlich weiter verbreitet, jedoch auch nicht für alle Menschen zugänglich: Fast zwei Milliarden Menschen besitzen kein Mobiltelefon und eine halbe Milliarde haben in ihrem Wohnumfeld kein Mobilfunknetz (ebd.). Soziale Medien wie zum Beispiel Facebook und Messengerdienste wie WhatsApp werfen die Frage auf, ob durch sie weitere Möglichkeiten der digitalen Kommunikation dazugewonnen wurden. Eine weitere Nutzung kann hier zum Beispiel der Aufbau eines individuellen digitalen Netzwerks, zur Kommunikation mit bekannten und unbekannten Menschen über gemeinsame Interessen, sein. Die Teilhabe an den sozialen Medien ist jedoch noch nicht für alle Menschen möglich, knapp vier Fünftel der Weltbevölkerung sind noch in keinem sozialen Netzwerk registriert (ebd., S. 27). Der Austausch von Fotos und Videos sowie Text- und Sprachnachrichten und Möglichkeiten des internetbasierten Telefonierens über die diversen Apps ist heute scheinbar einfach machbar. $\mathrm{Ob}$ zum einen die Kommunikation hiermit niederschwellig zugäng- 
lich ist und zum anderen die Intensität der Kommunikation über weite Entfernungen durch diese Möglichkeiten komplexer und höher wird, ist zu hinterfragen. Bedenklich ist, dass bei diversen Apps die Nutzung häufig nur scheinbar kostenlos funktioniert, da die „Bezahlung“ oftmals durch die Bereitstellung der eigenen Daten stattfindet (ebd., S. 44).

Bei den vielfältigen Anwendungen der Digitalisierung wird als eine große Gemeinsamkeit immer wieder das Potential der Skalierung genannt. Mit einer Skalierung wird angestrebt, dass eine Verbreitung eines Angebots erreicht werden kann ohne den Aufwand wesentlich zu erhöhen. Skalierung bedeutet damit, dass bei einer ansteigenden Zahl an Nutzern, der Einsatz von Ressourcen annähernd gleich bleibt. Somit kann eine hohe Effizienz in Aussicht gestellt werden. Ein Beispiel hierfür ist die Bereitstellung von Informationen auf digitalen Plattformen. Ihnen wird das Potential zugeschrieben, dass es keinen Unterschied macht, wie viele Menschen die Informationen auf der Plattform nutzen, da der Einsatz von Ressourcen bei mehr Nutzern häufig nicht viel größer wird. Analoge Verbreitungswege von Informationen zu nutzen, beispielsweise mit Hilfe von Büchern, werden hingegen oftmals als deutlich aufwendiger und kostenintensiver angesehen. Ausgehend von den geringeren Kosten für die Transaktion von digitalen Informationen bietet es sich an, die potentiellen finanziellen Vorteile durch die erhöhte Effizienz für Unternehmen, Regierungen und die Bevölkerung zu untersuchen (The World Bank, 2016b, S. 1). Problematisch kann sein, dass sich der Effekt der Skalierung und damit der einfachen Verbreitung von Informationen sowohl bei den Vorteilen der Digitalisierung als auch bei den Risiken und Gefahren wiederfindet.

\section{Digitalisierung in der \\ Entwicklungszusammenarbeit}

Es ist strittig, welchen Stellenwert die Digitalisierung in der deutschen Entwicklungszusammenarbeit einnimmt. Beispielsweise unterstreicht Bundesentwicklungsminister Gerd Müller die Potentiale, die mit der Digitalisierung aus seiner Sicht einhergehen: „Die 17 Ziele für nachhaltige Entwicklung (SDGs) können wir bis zum Jahr 2030 nur erreichen, wenn wir es schaffen, die entwicklungspolitischen Chancen der Digitalisierung weltweit nutzbar zu machen" (Müller, 2017a, S. 9). Über die politische Ebene hinaus stellt sich die Frage, inwieweit digitale Medien in Nichtregierungsorganisationen (NRO) bereits genutzt werden. Nach Sangmeister sind heute schon neue Informations- und Kommunikationstechnologien in der deutschen Entwicklungszusammenarbeit gängig und etabliert (2018, S. 22).

Der viel diskutierte Begriff Entwicklung kann im Zuge der Digitalisierung eine weitere Dimension bekommen: Es ist zu klären, ob neben der ökonomischen, sozialen und politischen Entwicklung eines Landes nun auch die digitale Entwicklung eine bedeutsame Rolle einnimmt. Darüber hinaus ist eine offene Frage, inwieweit die digitale Entwicklung eines Landes direkt mit den ökonomischen, sozialen und politischen Entwicklungen zusammenhängt und diese sich gegenseitig beeinflussen. Beispielsweise könnte für alle Entwicklungen die Neuheit, dass bislang einige etablierte Geschäftsmodelle durch digitale Lösungen abgelöst werden, eine Relevanz haben. Hier kann von einer möglichen Disruption gesprochen werden, d.h. von der Verdrängung alter Geschäftsmodelle vom Markt durch neue innovative Alternativen. Da die innovativen, digitalen Alternativen von der bereits erwähnten Skalierung profitieren können und somit mit geringen Ressourcen viele Nutzer potentiell erreichen können, wird bei dem Aufbau eines solchen Geschäftsmodells oftmals als großer Vorteil gesehen, dass nicht unbedingt ein großes Startkapital notwendig ist, um erfolgreich zu sein. Durch diesen Vorteil stellt sich die Frage, ob gerade in diesen innovativen, digitalen Geschäftsmodellen für Menschen, denen wenige Ressourcen zur Verfügung stehen, eine Chance zum Aufbau eines erfolgreichen Unternehmens besteht. Strittig ist, ob perspektivisch hierdurch ein Zugang für einige Menschen in Ländern des Globalen Südens zu Märkten möglich wird, die bislang für sie verschlossen waren. Dieser Zugang kann jedoch nur möglich werden, wenn Menschen in Ländern des Globalen Südens Zugang zu Bildung, digitalen Kompetenzen und einer digitalen Infrastruktur haben. Sangmeister folgend sehen Experten in einigen Ländern des Globalen Südens darüber hinaus das Potential durch Digitalisierung technische Entwicklungen zu überspringen und so den Stand der Länder des Globalen Nordens in manchen Bereichen aufzuholen oder sogar zu überholen, wie es zum Beispiel im Bereich des digitalen Zahlungsverkehrs bereits geschieht (2018, S. 23f.).

Über die Potentiale für unternehmerische Erfolge in Ländern des Globalen Südens hinaus wird diesen neuen Geschäftsmodellen bereits heute vermehrt eine Relevanz für unsere globale Gesellschaft zugesprochen: Sie sind weltweit immer häufiger erfolgreich, prägen bereits unsere Gesellschaft und schaffen neue Umstände in denen wir leben und wirtschaften, wohingegen die Diskussionen zu Werten und juristischen Fragestellungen rund um die Digitalisierung in vielerlei Hinsicht noch offen sind (Gimpel, 2018, S. 57).

Fraglich ist, wie die Organisationen der Entwicklungszusammenarbeit die Länder des Globalen Südens bei den diversen neuen digitalen Möglichkeiten und Herausforderungen unterstützen und entsprechende gemeinsame Programme aufbauen. Grundsätzlich sind beim Einsatz von digitalen Technologien sowohl ethische als auch Fragen der Wirksamkeit zu beachten. So zeichnet Martin-Shields (2017, S. 3f.) drei Risiken beim Einsatz der neuen digitalen Möglichkeiten auf. Er bezieht diese Risiken insbesondere auf Geflüchtete. Eine Übertragung auf die Zielgruppen der Entwicklungszusammenarbeit wird im Folgenden vorgenommen. Die drei Risiken in Bezug auf Angebote der Entwicklungszusammenarbeit beinhalten:

1. Ein Risiko ist, dass der Einsatz der Technologie um ihrer selbst willen geschieht, nur um die neue Technik auszuprobieren. Die Nutzung sollte vielmehr immer durch den Bedarf der jeweiligen Zielgruppe, die eine entsprechende Unterstützung oder Förderung erhalten soll, motiviert sein. Gegebenenfalls sind einfache Technologien hilfreicher als neue komplexe Anwendungen, weil sie beispielsweise niederschwelliger nutzbar sind (ebd.).

2. Eine Unterschätzung der finanziellen und personellen Ressourcen sowie der notwendigen Kompetenzen, die bei der Planung, Erarbeitung und Umsetzung digitaler Projekte häufig gebraucht werden, ist ein weiteres Risiko. Zudem ist es nicht einfach, digital-soziale Angebote zu entwickeln, die die Zielgruppe erreichen und ihnen einen individuellen Mehrwert bringen. Es sind entsprechende Fähigkeiten und Erfahrungswerte zur Entwicklung notwendig. Sowohl 
mangelnde Ressourcen als auch fehlende Fähigkeiten können einzelne NRO gegebenenfalls bei der Entwicklung digitaler Lösungen überfordern. Es gilt im Einzelfall zu diskutieren, ob beispielsweise IT-Experten mit ihren technischen Kompetenzen Unterstützung leisten können, damit die eigenen Pläne erfolgreich umgesetzt werden können (ebd.).

3. Die Verantwortung zum Schutz von persönlichen Daten und dem sicheren Gebrauch von digitalen Medien bei vulnerablen Gruppen liegt beim Anbieter der Technologien und kann eine besonders große Herausforderung darstellen. Für viele ethische und rechtliche Fragestellungen müssen Lösungen gefunden werden, damit Datenschutzvorschriften und Sorgfaltspflichten eingehalten werden (ebd.).

Der Einsatz von Technologien um ihrer selbst willen, die Unterschätzung der notwendigen Ressourcen und die Vernachlässigung von Datenschutz und Sorgfaltspflichten sind demnach drei Risiken, die bei der Einführung digitaler Technologien zu berücksichtigen sind. Die Vermeidung der aufgelisteten Risiken für NRO können erste Anhaltspunkte während des Aufbaus ihrer digitalen Aktivitäten sein. Zudem ist zu prüfen, ob bei der Auswahl digitaler Technologien die Kenntnis darüber, welche digitalen Medien und Technologien bereits durch die Zielgruppe genutzt werden und wie sie diese nutzen, Aufschluss darüber geben, in welche Richtung Projekte für sie entwickelt werden sollten. Innovationen, die durch die Zielgruppe selbst entstehen, bedürfen zudem gegebenenfalls nur eine finanzielle und technische Förderung und sind inhaltlich im besten Fall bereits bedarfsgerecht und wirkungsorientiert gestaltet (ebd., S. 4).

\section{Digitale Bildungsprojekte} in der Entwicklungszusammenarbeit

Für Bildung ist Kommunikation ein entscheidender Faktor, der Lernen ermöglicht und unterstützt. Es ist offen, welche Chancen und Herausforderungen die neuen digitalen Kommunikationsmöglichkeiten für die Bildungsvermittlung eröffnen. Zudem wird aktuell diskutiert, welchen Stellenwert die Bildung in der Entwicklungszusammenarbeit hat/haben soll und ob sich die Rolle der Bildung durch die Digitalisierung verändert.

Bundesentwicklungsminister Gerd Müller betont in seinem Buch „UNFAIR! Für eine gerechte Globalisierung“ (2017b), dass Bildung für die Entwicklungszusammenarbeit eine Schlüsselaufgabe darstellt. Er formuliert als politisches Ziel, dass in Entwicklungsländern mehr Mittel in Bildung investiert werden sollen. Deutschland arbeitet im Bereich der Bildung mit mehr als 40 Ländern zusammen, von denen bei zehn ein Fokus in der Zusammenarbeit auf das Thema Bildung gesetzt wurde (Müller, 2017b, S. 114).

Zur Rolle von Bildungsprojekten und Bildungsförderung in der Entwicklungszusammenarbeit wurde im November 2017 die Studie „Education first! Bildung entscheidet über die Zukunft Sahel-Afrikas“ vom Berlin-Institut für Bevölkerung und Entwicklung veröffentlicht. Sie stellt heraus, weshalb Bildung ein Schwerpunkt der deutschen Entwicklungszusammenarbeit sein sollte. Laut der Studie sollte - wie immer wieder diskutiert - nicht die Wirtschaftsförderung, die die deutsche Bundesregierung fokussiert, der Schwerpunkt der deutschen Entwicklungszusammenarbeit sein, sondern vielmehr die Bil- dung. Hier wird betont, dass Bildung die Basis für nachhaltiges Wirtschaftswachstum und eine langfristige Verbesserung der sozialen Lebenssituation der Bevölkerung und damit Bildungsförderung entscheidend für den Fortschritt eines Landes sei. Asiatische Länder, deren wirtschaftlicher und sozialer Aufstieg deutlich schneller vorangeschritten ist als in bezüglich des Lebensstandards vergleichbaren afrikanischen Ländern, zeichnen sich durch ein deutlich höheres Bildungsniveau der Bevölkerung aus. Dieser Zusammenhang kann ebenfalls ein Hinweis auf die Bedeutung der Bildung für die Entwicklung eines Landes sein (Klingholz, 2017, S. 2). Eine besondere Herausforderung in der Förderung durch Bildung stellt zum einen die lange Dauer bis sich Ergebnisse zeigen dar. Es vergehen viele Jahre, bis ein Beruf aufgenommen wird und sowohl das Individuum als auch der Staat und die Gesellschaft von der Investition in die Bildung profitieren. Zum anderen ist besonders herausfordernd, dass der Aufbau einer Bildungsinfrastruktur ein starkes Bevölkerungswachstum, beispielsweise in Subsahara Afrika, berücksichtigen müsste (ebd., S. 2f.). Für die zweite Herausforderung ist zu diskutieren, welchen Mehrwert die Digitalisierung durch die Chance der Skalierbarkeit bieten kann. Viele Menschen zu erreichen und dabei ressourcensparend vorzugehen, ist wie bereits beschrieben, ein Potenzial verschiedener digitaler Tools, daher gilt es hier diese Möglichkeit auch in Bezug auf Bildung zu überprüfen. Zum Beispiel können Menschen, die in Ländern des Globalen Südens leben, durch Handys und Internetempfang niederschwellig Zugang zu Informationen und Wissen bekommen, was zur Inklusion in einigen Lebensbereichen führen kann. Voraussetzung hierfür ist beispielsweise, dass sowohl die Infrastruktur für einen Internetzugang durch die Regierung ermöglicht werden muss und beispielsweise durch Subventionen Handys für die breite Bevölkerung erschwinglich werden. Zugang zu Wissen und Informationen kann, nach der Schaffung dieser und weiterer Grundlagen, einfach und kostengünstig möglich werden (The World Bank, 2016b, S. 1).

Fraglich ist, wie die Rolle von Bildung in Zukunft global aussehen wird. Dabei steht zur Diskussion, ob zum Beispiel die Ausbildung für einen konkreten Beruf sowohl in Ländern des Globalen Nordens als auch in Ländern des Globalen Südens für die gesamte Zeit im Berufsleben eines Menschen ausreichend sein wird, um den sich immer wieder verändernden Arbeitsbedingungen und neuen Aufgaben gerecht zu werden. Von diesen zukünftig häufigen Veränderungen im Arbeitsleben wird auf Grund der sich rasant wandelnden digitalen Technologien ausgegangen. Um diesen Umständen zu begegnen, gilt es zu klären, ob und in welcher Hinsicht Kompetenzen über eine singuläre Berufsausbildung hinaus notwendig sein werden und welche Fortbildungen im Laufe einer Berufslaufbahn hilfreich sind. Dementsprechend brauchen die Arbeitnehmer der Zukunft eine Förderung, die auf die neuen Anforderungen des sich ständig weiterentwickelnden Arbeitsmarktes vorbereiten (ebd., S. 3). Beispielsweise geht die Weltbank davon aus, dass die Förderung des lebenslangen Lernens in der Entwicklungszusammenarbeit zukünftig an Bedeutung gewinnt (ebd., S. 4). Ausgehend von der Notwendigkeit, in der Lage zu sein, sich auf neue berufliche Herausforderungen immer wieder anzupassen, ist zu prüfen, inwieweit dabei soziale und kognitive Kompetenzen sowie Flexibilität und die Bereitschaft zur eigenen Weiterentwicklung hilfreich sein können. Die Relevanz der Förderung sozialer 
Kompetenzen sieht Bundesentwicklungsminister Gerd Müller und schließt neben der formalen Bildung unter anderem auch die emotionalen Kompetenzen als entscheidender Förderbereich mit ein (Müller, 2017b, S. 90). Zu sozialen Kompetenzen kann „die Fähigkeit zu kritischem Denken, zur Lösung von Problemen und Konflikten, also um Voraussetzungen für die Entwicklung zu einem verantwortungsvollen Weltbürger" (Klingholz, 2017, S. 3) gehören. Es ist fraglich, ob sich die Vermittlung sozialer Kompetenzen über digitale Medien schwieriger oder einfacher als in der analogen Bildung gestaltet.

Digitalisierungsprojekte, auch im Bereich der Bildung, können ebenfalls eine analoge Komponente beinhalten und damit ihre Zielgruppen sowohl digital als auch analog ansprechen. Mit diesem Vorgehen wird häufig angestrebt, online verfügbare Angebote durch eine analoge Bewerbung zu verbreiten. Zum Beispiel können Multiplikatoren versuchen, neben digitalen auch über analoge Wege potentielle Nutzer anzusprechen, sie für das Angebot zu gewinnen und in einem nächsten Schritt daran $\mathrm{zu}$ binden. Es ist $\mathrm{zu}$ untersuchen, ob Bildungsangebote von einem hybriden System, d.h. von einer Kombination von analog und digital, auch inhaltlich profitieren können oder beide Wege zu nutzen vielmehr eine Verschwendung von Ressourcen darstellt. Grundsätzlich stellt sich die Frage, ob durch die beschriebene Skalierbarkeit digitaler Angebote, diese im Vergleich zur analogen Arbeit effizienter sind oder auch analoge Angebote gleichsam effizient vorgehen können. Eine große Chance wird in hybriden Angeboten hinsichtlich der Integration von Menschen ohne Internetzugang oder ohne digitale Kompetenzen gesehen. Da sie von digitalen Angeboten ausgeschlossen sein können, wären analoge Ergänzungsangebote so auszurichten, dass sie auch diese Zielgruppe partizipieren lassen. Dabei kann eine Prüfung, ob ein Zugang zu den digitalen Angeboten durch Unterstützung möglich gemacht werden kann, eine weitere Aufgabe analoger Ergänzungsangebote sein (Breidenbach et al., 2014, S. 25ff.).

Es besteht das Problem, dass sozial-digitale Angebote immer wieder ungenutzt bleiben. Gründe hierfür können zum Beispiel sein, dass die Angebote die gewünschte Zielgruppe nicht erreichen, die möglichen Nutzeranwendungen nicht den Bedürfnissen der Adressaten entsprechen oder die potentiellen Nutzer nicht über die notwendigen Voraussetzungen zur Nutzung verfügen, wie beispielsweise der bereits erwähnte fehlende Zugang zum Internet oder die mangelnden digitalen Kompetenzen. Daher sind der Austausch mit der Zielgruppe und die Involvierung potentieller Nutzer bei der Entwicklung digitaler Angebote und Anstrengungen zur Erreichung der Zielgruppe in vielen Fällen notwendig, damit digitale Angebote ihren Zweck erfüllen können (ebd.).

\section{Ausblick}

Digitale Technologien bringen eine Vielzahl an komplexen Fragestellungen und Risiken mit sich, haben jedoch auch das Potential innovative und skalierbare Lösungen für soziale Probleme und Bildungsfragen zu bieten (Martin-Shields, 2017, S. 4). Damit auch Länder des Globalen Südens von den neuen Möglichkeiten profitieren können, müssen zunächst die analogen Voraussetzungen bereitgestellt werden, allen voran der $\mathrm{Zu}$ - gang zum Internet. Zudem müssen die rechtliche Rahmenbedingungen sowie breite Fortbildungsmöglichkeiten bezüglich digitaler Kompetenzen geschaffen werden (The World Bank, 2016b, S. 1).

Die Bildungsfrage ist zum einen so entscheidend und zum anderen so herausfordernd für die Zukunft afrikanischer Länder, so dass Unterstützung von außen notwendig erscheint. Dies wird dadurch verstärkt, dass sich die Entwicklungen in einzelnen Ländern global auswirken und im Kontext von Migrationsbewegungen Auswirkungen auf Europa und Deutschland haben können (Klingholz, 2017, S. 2f.). Als noch bedeutsamer erscheinen jedoch die Potentiale und Chancen, die Sustainable Development Goals der Vereinten Nationen zu erreichen, wenn Menschen gefördert und ihre Kompetenzen zur Erreichung dieser Ziele eingesetzt würden. Zudem besteht das Menschenrecht "Jeder Mensch hat das Recht auf Bildung." (Allgemeine Erklärung der Menschenrechte (1948), Artikel 26), zu dessen Realisierung alle Möglichkeiten genutzt werden sollten, auch die Digitalen.

\section{Literatur}

gut.org gemeinnützige Aktiengesellschaft betterplace lab (Hrsg.) (2017). trendradar_2030. Ein Blick in die Zukunft der digitalen Technologien und wie sie unsere Welt besser machen können. Berlin.

Breidenbach, J., Mason, B., Prahm, M., Ullrich, A. \& Ziemann, K. (2014). Trendreport 2014. Berlin.

Gimpel, L. (2018). Doing Development Differently: Die digitale Transformation des Bundesunternehmens Deutsche Gesellschaft für Internationale Zusammenarbeit (GIZ). In H. Sangmeister \& H. Wagner (Hrsg.), Entwicklungszusammenarbeit 4.0 Digitalisierung und globale Verantwortung (S. 57-66). Baden-Baden: Nomos.

Kaps, A. \& Reinig, A. \& Müller, R. \& Klingholz, R. (2017). Education first! Bildung entscheidet über die Zukunft Sahel-Afrikas. Zugriff am 06.07.2018 https://www.ber lin-institut.org/publikationen/studien/education-first.html

Klingholz, R. (2017). Ohne Bildung keine Perspektive. In Berlin-Institut für Bevölkerung und Entwicklung (Hrsg.), Education first! Bildung entscheidet über die Zukunft Sahel-Afrikas. (S. 2-3). Zugriff am 06.07.2018 https://www.berlin-institut.org/publi kationen/studien/education-first.html

Martin-Shields, C.-P. (2017). Digitalisierung und Flucht: Wie können Geber digitale Technologien zur Unterstützung von Flüchtlingen einsetzen? Zugriff am 06.07.2018 https://www.die-gdi.de/uploads/media/AuS_16.2017.pdf

Müller, G. (2017a). Wie digital ist die EZ? In gut.org gemeinnützige Aktiengesellschaft, betterplace lab (Hrsg.). trendradar_2030. Ein Blick in die Zukunft der digitalen Technologien und wie sie unsere Welt besser machen können (S. 8-11). Berlin.

Müller, G. (2017b). Unfair! Für eine gerechte Globalisierung. Hamburg: Murmann Publishers $\mathrm{GmbH}$.

Sangmeister, H. (2018). EZ 4.0 - Chancen, Risiken und viele offene Fragen. In H. Sangmeister \& H. Wagner (Hrsg.), Entwicklungszusammenarbeit 4.0-Digitalisierung und globale Verantwortung (S. 21-32). Baden-Baden: Nomos. https://doi. org/10.5771/9783845290560-21

Schönig, W., Hoyer, T. \& Potratz, A. (2018). Lehrbuch Ökonomie in der Sozialen Arbeit. Köln: Beltz Juventa.

The World Bank (2016a). Digital Dividends. World Development Report 2016. Zugriff am 06.07.2018 http://www.worldbank.org/en/publication/wdr2016

The World Bank (2016b). Kernbotschaften. Digitale Dividenden. Weltentwicklungsbericht 2016. Zugriff am 06.07.2018 http://pubdocs.worldbank.org/en/ 112781453827891613/WDR-2016-MainMessages-GERMAN-Final.pdf

\section{Alexandra Potratz, Dipl. Soz.Arb./Soz.Päd., M.A.} Sozialmanagement

ist seit 2016 wissenschaftliche Mitarbeiterin an der Katholischen Hochschule NRW und in diesem Rahmen für die Internationalisierung der Hochschule im Kontext der Entwicklungszusammenarbeit zuständig. Sie ist Gründerin und Vorstandsvorsitzende des intombi e.V. und promoviert an der Universität zu Köln zu dem Thema "Online neue Wege gehen in der Entwicklungszusammenarbeit“. Sie publizierte zuletzt gemeinsam mit Prof. Werner Schönig und Thomas Hoyer das Lehrbuch "Ökonomie in der Sozialen Arbeit". 